\title{
A NOTE ON EQUILIBRATED STRESS FIELDS FOR NO-TENSION BODIES UNDER GRAVITY
}

\author{
BY \\ M. LUCCHESI (Dipartimento di Costruzioni, Università di Firenze, Piazza Brunelleschi 6, 50121 \\ Firenze, Italia), \\ M. ŠILHAVÝ (Dipartimento di Matematica, Università di Pisa, Largo Bruno Pontecorvo 5, 56127 \\ Pisa, Italia), \\ AND
}

N. ZANI (Dipartimento di Costruzioni, Università di Firenze, Piazza Brunelleschi 6, 50121 Firenze, Italia)

\begin{abstract}
We study the equilibrium problem for two-dimensional bodies made of a no-tension material under gravity, subjected to distributed or concentrated loads on their boundary. Admissible and equilibrated stress fields are interpreted as tensor-valued measures with distributional divergence represented by a vector-valued measure, as developed by the authors of the present paper. Such stress fields allow us to consider stress concentrations on surfaces and lines. Working in $\mathbb{R}^{n}$, we calculate the weak divergence of a stress field that is asymptotically of the form $|\boldsymbol{x}|^{-n+1} \boldsymbol{T}_{0}(\boldsymbol{x} /|\boldsymbol{x}|)$ for $\boldsymbol{x} \rightarrow \mathbf{0}$ on a region that is asymptotically a cone with vertex $\mathbf{0}$. Such stress fields arise as parts of our solutions for two-dimensional panels. Proceeding to problems in dimension two, we first determine an admissible equilibrated solution for a half-plane under gravity that underlies two subsequent solutions for rectangular panels. For the latter we give solutions for three types of loads.
\end{abstract}

1. Introduction. We study the equilibrium problem of bodies made of a no-tension (or masonry-like) material [1], [3] under gravity. We seek stress fields that are equilibrated with the applied loads and compatible with the incapability of the material to withstand traction. Referring to [10] for a motivation in the context of limit analysis [2],

Received February 3, 2006.

2000 Mathematics Subject Classification. Primary 74G70; Secondary 49Q15.

Key words and phrases. Masonry panels, equilibrium, divergence measures.

The authors thank the referee for helpful comments on the previous version of the paper. The research of M. Šilhavý was supported by a grant of MIUR "Variational theory of microstructure, semiconvexity, and complex materials." The support is gratefully acknowledged.

E-mail address: massimiliano.lucchesi@unifi.it

Current address: Mathematical Institute of the AV ČR, Žitná 25, 11567 Prague 1, Czech Republic.

E-mail address: silhavy@math.cas.cz

E-mail address: nicola.zani@unifi.it 
we observe that these solutions can be used to determine lower bounds for the collapse load and sometimes the collapse load itself. The solution to this problem is considerably simplified by allowing for singularities of the stress field on one or more surfaces or curves of concentrated stress. This feature corresponds to infinite compressive strength of the material, a simplifying hypothesis that is frequently used in the study of masonry structures [6]. As in [7], [8], [9], [10], we use tensor-valued measures to describe the singular stress fields. We refer to [11], [12], [13] for solutions for two-dimensional panels in the absence of gravity and to [9] for some three-dimensional solutions.

In the present paper we consider two-dimensional rectangular panels under gravity and several types of loads. We first review the basic facts on stresses interpreted as measures in the general setting in $\mathbb{R}^{n}$ and calculate the weak divergence and the normal trace of a stress field that is asymptotically of the form $|\boldsymbol{x}|^{-n+1} \boldsymbol{T}_{0}(\boldsymbol{x} /|\boldsymbol{x}|)$ on a region that is asymptotically a cone with vertex $\mathbf{0}$ as $\boldsymbol{x} \rightarrow \mathbf{0}$. Such stress fields arise as parts of our solutions for two-dimensional panels. Proceeding to dimension two, we first give a solution for a half-plane under gravity and prescribed distributed loads on its boundary. The latter underlies two solutions subsequently given for rectangular panels. The rectangular panels are subjected to three types of loads, and the solutions are glued from solutions on various subregions of the panel dictated by the load.

2. Divergence measure tensor fields. Throughout the paper, Lin denotes the space of all linear transformations from $\mathbb{R}^{n}$ into $\mathbb{R}^{n}$ with the scalar product $\boldsymbol{A} \cdot \boldsymbol{B}=$ $\operatorname{tr}\left(\boldsymbol{A} \boldsymbol{B}^{\top}\right), \boldsymbol{A}, \boldsymbol{B} \in$ Lin, and Sym is a subspace of Lin consisting of all symmetric transformations. We interpret Lin as the space of all second order tensors and use vector and tensor notation and conventions from [5], [16].

If $\Omega$ is an open subset of $\mathbb{R}^{n}$, we denote by $C_{0}^{\infty}\left(\Omega, \mathbb{R}^{n}\right)$ the set of all infinitely differentiable functions $\boldsymbol{v}: \mathbb{R}^{n} \rightarrow \mathbb{R}^{n}$ whose support spt $\boldsymbol{v}$ is contained in $\Omega$.

Throughout the paper, we use Lin-valued measures on $\mathbb{R}^{n}$ to describe fields of the stress tensor in a body and $\mathbb{R}^{n}$-valued measures on $\mathbb{R}^{n}$ to describe body forces acting on the body or surface tractions acting on the boundary of the body. These measures are $\sigma$ additive functions $\boldsymbol{\mu}$ with values in Lin or in $\mathbb{R}^{n}$ defined on the collection of all Borel subsets of $\mathbb{R}^{n}$. If $A \subset \mathbb{R}^{n}$ is a Borel set, we say that $\boldsymbol{\mu}$ is supported by $A$ if $\boldsymbol{\mu}(B)=\mathbf{0}$ for each Borel set $B \subset \mathbb{R}^{n}$ with $A \cap B=\emptyset$; equivalently, we say that $\boldsymbol{\mu}$ vanishes outside $A$. We denote by $\mathcal{M}(A, \operatorname{Lin})$ [or by $\mathcal{M}\left(A, \mathbb{R}^{n}\right)$ ] the set of all Lin-valued [or $\mathbb{R}^{n}$-valued] measures on $\mathbb{R}^{n}$ that vanish outside $A$. Thus our convention is that all measures are defined on the whole of $\mathbb{R}^{n}$, but we shall often work with measures supported by one or another subset of $\mathbb{R}^{n}$.

If $\Omega \subset \mathbb{R}^{n}$ is open, we say that $\mathbf{T} \in \mathcal{M}(\Omega, \operatorname{Lin})$ is a divergence measure tensor field in $\Omega$ if there exists a measure $\operatorname{div} \mathbf{T} \in \mathcal{M}\left(\Omega, \mathbb{R}^{n}\right)$, called the divergence of $\mathbf{T}$ in $\Omega$, such that

$$
\int_{\Omega} \nabla \boldsymbol{v} \cdot d \mathbf{T}=-\int_{\Omega} \boldsymbol{v} \cdot d \mathbf{d i v} \mathbf{T}
$$


for each $\boldsymbol{v} \in C_{0}^{\infty}\left(\Omega, \mathbb{R}^{n}\right)$. A measure $\mathbf{T} \in \mathcal{M}(\Omega$, Lin $)$ is said to be an equilibrated tensor field in $\Omega$ if there exist measures $\mathbf{b} \in \mathcal{M}\left(\Omega, \mathbb{R}^{n}\right)$ and $\mathbf{t} \in \mathcal{M}\left(\partial \Omega, \mathbb{R}^{n}\right)$ such that

$$
\int_{\Omega} \nabla \boldsymbol{v} \cdot d \mathbf{T}=\int_{\Omega} \boldsymbol{v} \cdot d \mathbf{b}+\int_{\partial \Omega} \boldsymbol{v} \cdot d \mathbf{t}
$$

for each $\boldsymbol{v} \in C_{0}^{\infty}\left(\mathbb{R}^{n}, \mathbb{R}^{n}\right)$. Here $\partial \Omega$ denotes the topological boundary of $\Omega$. Since the measures $\mathbf{b}$ and $\mathbf{t}$ are supported on the disjoint sets $\Omega$ and $\partial \Omega$, respectively, they are uniquely determined (provided they exist). We call the pair $(\mathbf{b}, \mathbf{t})$ the $\mathbf{l o a d}$ corresponding to $\mathbf{T}$ and the measure $\mathbf{t}$ the normal trace of $\mathbf{T}$ on $\partial \Omega$; we use the notation $\mathrm{N}(\mathbf{T}):=\mathbf{t}$ for the normal trace. Equation (2.1) then reads

$$
\int_{\Omega} \nabla \boldsymbol{v} \cdot d \mathbf{T}=-\int_{\Omega} \boldsymbol{v} \cdot d \operatorname{div} \mathbf{T}+\int_{\partial \Omega} \boldsymbol{v} \cdot d \mathrm{~N}(\mathbf{T})
$$

for each $\boldsymbol{v} \in C_{0}^{\infty}\left(\mathbb{R}^{n}, \mathbb{R}^{n}\right)$. Clearly, any equilibrated tensor field $\mathbf{T}$ is a divergence measure tensor field and if $(\mathbf{b}, \mathbf{t})$ is the load, then $\operatorname{div} \mathbf{T}=-\mathbf{b}$. We refer to [10] for the literature. There are divergence measure tensor fields that are not equilibrated [17, Example 9.1], i.e., for which the measure $\mathbf{t}$ does not exist.

We denote by $\mathcal{L}^{n}$ the Lebesgue measure in $\mathbb{R}^{n}\left[4\right.$, Subsection 2.6.5] and by $\mathcal{H}^{k}$ the $k$-dimensional Hausdorff measure [4, Subsections 2.10.2-2.10.6]. We denote by $\delta_{\mathbf{0}}$ the scalar Dirac measure at $\mathbf{0}$. If $\boldsymbol{\mu}$ is a measure in $\mathbb{R}^{n}$ and $B \subset \mathbb{R}^{n}$ a Borel set, we denote by $\boldsymbol{\mu} L B$ the restriction of $\boldsymbol{\mu}$ to $B$, i.e., a measure on $\mathbb{R}^{n}$ defined by

$$
(\boldsymbol{\mu}\llcorner B)(A)=\boldsymbol{\mu}(A \cap B)
$$

for any Borel subset $A$ of $\mathbb{R}^{n}$. If $f$ is a Borel $\boldsymbol{\mu}$ integrable function, defined $\boldsymbol{\mu}$ almost everywhere, we denote by $f \boldsymbol{\mu}$ the product of $f$ and $\boldsymbol{\mu}$, i.e., the measure defined by

$$
(f \boldsymbol{\mu})(A)=\int_{A} f d \boldsymbol{\mu}
$$

for every Borel set $A \subset \mathbb{R}^{n}$. Note that $(f \boldsymbol{\mu})\llcorner B=f(\boldsymbol{\mu}\llcorner B)$ and we use the symbol $f \boldsymbol{\mu}\llcorner B$ for the last two expressions.

If an equilibrated tensor field $\mathbf{T} \in \mathcal{M}(\Omega, \operatorname{Lin})$ is interpreted as the stress field in a continuous body under the action of a body force given by a prescribed measure $\mathbf{b}_{0} \in$ $\mathcal{M}\left(\Omega, \mathbb{R}^{n}\right)$ and the boundary traction given by a prescribed measure $\mathbf{t}_{0} \in \mathcal{M}\left(\partial \Omega, \mathbb{R}^{n}\right)$, then the equations of equilibrium read

$$
\begin{gathered}
\operatorname{div} \mathbf{T}+\mathbf{b}_{0}=\mathbf{0}, \\
\mathrm{N}(\mathbf{T})=\mathbf{t}_{0} .
\end{gathered}
$$

In particular, if $\mathbf{b}_{0}$ is absolutely continuous with respect to the Lebesgue measure (e.g., the gravity), i.e.,

$$
\mathbf{b}_{0}=\boldsymbol{b}_{0} \mathcal{L}^{n}\llcorner\Omega
$$

where $\boldsymbol{b}_{0}: \Omega \rightarrow \mathbb{R}^{n}$ is an $\mathcal{L}^{n}$ integrable function, then $\operatorname{div} \mathbf{T}$ must be absolutely continuous with respect to $\mathcal{L}^{n}$ as well.

More generally, assume that $\mathbf{T}$ consists of a regular part $\boldsymbol{T}_{r}$ distributed over $\Omega$ and of a singular part $\boldsymbol{T}_{s}$ concentrated on an $(n-1)$-dimensional oriented surface $\mathcal{S}$, i.e.,

$$
\mathbf{T}=\boldsymbol{T}_{r} \mathcal{L}^{n} \mathrm{~L} \Omega+\boldsymbol{T}_{s} \mathcal{H}^{n-1} \mathrm{~L} \mathcal{S} .
$$


Assume that $\mathcal{S}$ has a piecewise smooth boundary $\partial \mathcal{S}$ that is contained in the boundary $\partial \Omega$ of $\Omega$, that the regular part $\boldsymbol{T}_{r}$ is piecewise continuously differentiable with a jump discontinuity $\left[\boldsymbol{T}_{r}\right]$ on $\mathcal{S}$ and that the singular part $\boldsymbol{T}_{s}$ is superficial and continuously differentiable in the interior of $\mathcal{S}$. The requirement that $\boldsymbol{T}_{s}$ be superficial means that $\boldsymbol{T}_{s}(\boldsymbol{x}) \boldsymbol{n}(\boldsymbol{x})=0$ for any $\boldsymbol{x} \in \mathcal{S}$ where $\boldsymbol{n}$ is the normal to $\mathcal{S}$. Under the assumptions of [10, Section 3(ii)] then (2.3) with (2.4) reduces to

$$
\operatorname{div} \boldsymbol{T}_{r}+\boldsymbol{b}_{0}=\mathbf{0} \quad \text { in } \quad \Omega \backslash \mathcal{S}
$$

and

$$
\left[\boldsymbol{T}_{r}\right] \boldsymbol{n}-\operatorname{div}_{\mathcal{S}} \boldsymbol{T}_{s}=\mathbf{0} \quad \text { on } \quad \mathcal{S}
$$

where div is the classical divergence operator and $\operatorname{div}_{\mathcal{S}}$ denotes the surface divergence. Moreover, if $\Omega$ is a region with Lipschitz boundary, then under the assumptions of $[10$, Section 3(ii)] we have

$$
\mathrm{N}(\mathbf{T})=\boldsymbol{T}_{r} \boldsymbol{m} \mathcal{H}^{n-1}\left\llcorner\partial \Omega+\boldsymbol{T}_{s} \boldsymbol{p} \mathcal{H}^{n-2}\llcorner(\partial \Omega \cap \partial \mathcal{S})\right.
$$

where $\boldsymbol{m}$ is the outer normal to $\partial \Omega$ and $\boldsymbol{p}$ is the outer normal to $\partial \mathcal{S}$.

A subset $C$ of $\mathbb{R}^{n}$ is said to be a cone with vertex at $\mathbf{0}$ if $r \boldsymbol{v} \in C$ for each $r>0$ and $\boldsymbol{v} \in C$. For each $r>0$ let $\mathbf{B}(r)$ denote the open ball in $\mathbb{R}^{n}$ of center $\mathbf{0}$ and radius $r$ and let $\overline{\mathbf{B}}(r)$ be the closure of $\mathbf{B}(r)$. We denote by cl $\Omega$ the closure of $\Omega \subset \mathbb{R}^{n}$ and let $\mathbb{S}^{n-1}$ be the unit sphere in $\mathbb{R}^{n}$.

The following proposition will be employed to determine the divergences and traces of (parts of) the singular stress fields of Section 3 and of those in Examples 1 and 3 in Section 4.

Proposition 2.1. Consider the following objects:

- a bounded region $\Omega$ with Lipschitz boundary in $\mathbb{R}^{n}$ of exterior normal $\boldsymbol{m}$,

- a region $W \subset \Omega$ with Lipschitz boundary of exterior normal $\boldsymbol{n}$ with $\mathbf{0} \in \partial W$,

- a tensor-valued continuous map $\boldsymbol{T}$ on $\mathrm{cl} W \backslash\{\mathbf{0}\}$, continuously differentiable in $W$, with $\operatorname{div} \boldsymbol{T}$ integrable on $W$ and $\boldsymbol{T} \boldsymbol{n}$ integrable on $\partial W$, satisfying $|\boldsymbol{T}(\boldsymbol{x})| \leq c|\boldsymbol{x}|^{-n+1}$ for some $c$ and every $\boldsymbol{x} \in W \backslash\{\mathbf{0}\}$,

and assume that there exists a cone $C$ with vertex $\mathbf{0}$ and a bounded $\mathcal{H}^{n-1}$ measurable function $\boldsymbol{T}_{0}: C \cap \mathbb{S}^{n-1} \rightarrow$ Lin such that if $\Delta(W, C)$ denotes the symmetric difference of $W$ and $C$, then

$$
\begin{gathered}
\lim _{r \rightarrow 0} \mathcal{H}^{n-1}[\Delta(W, C) \cap \partial \mathbf{B}(r)] / r^{n-1}=0, \\
\lim _{r \rightarrow 0} \sup \left\{\left|r^{n-1} \boldsymbol{T}(\boldsymbol{x})-\boldsymbol{T}_{0}(\boldsymbol{x} / r)\right|: \boldsymbol{x} \in C \cap \partial \mathbf{B}(r)\right\}=0 .
\end{gathered}
$$

Then

$$
\mathbf{T}:=\boldsymbol{T} \mathcal{L}^{n} \mathrm{~L} W
$$

is an equilibrated tensor field in $\Omega$; moreover, if $\mathbf{0} \in \Omega$, then

$$
\begin{gathered}
\operatorname{div} \mathbf{T}=\boldsymbol{c} \delta_{\mathbf{0}}+\operatorname{div} \boldsymbol{T} \mathcal{L}^{n}\left\llcorner W-\boldsymbol{T} \boldsymbol{n} \mathcal{H}^{n-1}\llcorner(\partial W \cap \Omega),\right. \\
\mathrm{N}(\mathbf{T})=\boldsymbol{T} \boldsymbol{m} \mathcal{H}^{n-1}\llcorner(\partial \Omega \cap \partial W)
\end{gathered}
$$


where

$$
c=-\int_{C \cap \mathbb{S}^{n-1}} \boldsymbol{T}_{0}(\boldsymbol{d}) \boldsymbol{d} d \mathcal{H}^{n-1}(\boldsymbol{d})
$$

while if $\mathbf{0} \in \partial \Omega$, then

$$
\begin{gathered}
\operatorname{div} \mathbf{T}=\operatorname{div} \boldsymbol{T} \mathcal{L}^{n}\left\llcorner W-\boldsymbol{T} \boldsymbol{n} \mathcal{H}^{n-1} \mathrm{~L}(\partial W \cap \Omega),\right. \\
\mathrm{N}(\mathbf{T})=-\boldsymbol{c} \delta_{\mathbf{0}}+\boldsymbol{T} \boldsymbol{m} \mathcal{H}^{n-1}\llcorner(\partial \Omega \cap \partial W) .
\end{gathered}
$$

This generalizes [10, Proposition 2.2] in which $W=\Omega \cap C$ with $C$ a cone and

$$
\boldsymbol{T}(\boldsymbol{x})=|\boldsymbol{x}|^{-n-1} \alpha(\boldsymbol{x} /|\boldsymbol{x}|) \boldsymbol{x} \otimes \boldsymbol{x},
$$

$\boldsymbol{x} \in(\operatorname{cl} C \cap \Omega) \backslash\{\boldsymbol{0}\}$ where $\alpha$ is a scalar-valued function. In accordance with our conventions on measures, $\mathbf{T}$ is a measure on $\mathbb{R}^{n}$ which vanishes outside $W$. In (2.12), $\boldsymbol{d}$ denotes the integration variable. The cone $C$ and the function $\boldsymbol{T}_{0}$ describe the asymptotics of the set $W$ and the field $\boldsymbol{T}$ near the singular point $\mathbf{0} \in \partial W$. Thus (2.7) says that the region $W$ looks asymptotically like $C$ near $\mathbf{0}$ while (2.8) says that $\boldsymbol{T}(\boldsymbol{x})$ is asymptotically equal to

$$
|\boldsymbol{x}|^{-n+1} \boldsymbol{T}_{0}(\boldsymbol{x} /|\boldsymbol{x}|)
$$

as $\boldsymbol{x} \rightarrow \mathbf{0}$; indeed, it is possible to show that $\mathrm{cl} C$ equals the tangent cone to $W$ at $\mathbf{0}[4$, Subsection 3.1.21] and

$$
\boldsymbol{T}_{0}(\boldsymbol{d})=\lim _{r \rightarrow 0} r^{n-1} \boldsymbol{T}(\mathbf{0}+r \boldsymbol{d})
$$

for every $\boldsymbol{d} \in C \cap \mathbb{S}^{n-1}$. It is easy to see that the hypotheses of the proposition guarantee that the individual terms in the right hand sides of (2.9), (2.10), (2.11), (2.13), (2.14) are well defined measures.

Proof. If $\boldsymbol{v} \in C_{0}^{\infty}\left(\mathbb{R}^{n}, \mathbb{R}^{n}\right)$, then

$$
\int_{\Omega} \nabla \boldsymbol{v} \cdot d \mathbf{T}=\int_{W} \nabla \boldsymbol{v} \cdot \boldsymbol{T} d \mathcal{L}^{n}=\lim _{r \rightarrow 0} \int_{W \backslash \overline{\mathbf{B}}(r)} \nabla \boldsymbol{v} \cdot \boldsymbol{T} d \mathcal{L}^{n} .
$$

Writing $W_{r}:=W \backslash \overline{\mathbf{B}}(r), S_{r}:=\partial \mathbf{B}(r), r>0$, we note that $W_{r}$ is a set of finite perimeter [4, Subsections 4.5.1-4] and for $\mathcal{L}^{1}$ a.e. $r>0$ the measure theoretic boundary $\partial W_{r}$ of $W_{r}$ is given by

$$
\partial W_{r}=(\partial W \backslash \overline{\mathbf{B}}(r)) \cup\left(W \cap S_{r}\right)
$$

to within a set of $\mathcal{H}^{n-1}$ measure 0 ; moreover, the measure theoretic normal $\boldsymbol{l}_{r}$ to $W_{r}$ is given by

$$
\boldsymbol{l}_{r}(\boldsymbol{x})= \begin{cases}\boldsymbol{n}(\boldsymbol{x}) & \text { on } \partial W \backslash \overline{\mathbf{B}}(r), \\ -\boldsymbol{x} / r & \text { on } W \cap S_{r}\end{cases}
$$

to within a change on a set of null $\mathcal{H}^{n-1}$ measure. To prove $(2.16)-(2.17)$, we note that $W_{r}=W \cap\left[\mathbb{R}^{n} \backslash \overline{\mathbf{B}}(r)\right]$ and use the integration by parts argument similar to that in the proof of [15, Proposition 2.4] with $M$ replaced by $W$ and $H_{t}$ replaced by $\mathbb{R}^{n} \backslash \overline{\mathbf{B}}(r)$; the details are omitted here. Formulas $(2.16)-(2.17)$ hold for $\mathcal{L}^{1}$ a.e. $r>0$; in the remaining 
part of the proof we consider only such values of $r$. We apply the divergence theorem for sets of finite perimeter [4, Subsection 4.5.6] and formulas (2.16)-(2.17) to obtain

$$
\begin{aligned}
\int_{W \backslash \overline{\mathbf{B}}(r)} \nabla \boldsymbol{v} \cdot \boldsymbol{T} d \mathcal{L}^{n}= & -\int_{W \backslash \overline{\mathbf{B}}(r)} \boldsymbol{v} \cdot \operatorname{div} \boldsymbol{T} d \mathcal{L}^{n} \\
& +\int_{\partial W \backslash \overline{\mathbf{B}}(r)} \boldsymbol{v} \cdot \boldsymbol{T} \boldsymbol{n} d \mathcal{H}^{n-1} \\
& -\int_{W \cap S_{r}} \boldsymbol{v} \cdot \boldsymbol{T}(\boldsymbol{x}) \boldsymbol{x} / r d \mathcal{H}^{n-1}(\boldsymbol{x}) .
\end{aligned}
$$

We denote the three integrals on the right hand side of (2.18) by $I_{r}^{1}, I_{r}^{2}, I_{r}^{3}$, respectively. Considering the limits $r \rightarrow 0$, we note that

$$
\begin{gathered}
I_{r}^{1} \rightarrow \int_{W} \boldsymbol{v} \cdot \operatorname{div} \boldsymbol{T} d \mathcal{L}^{n} \\
I_{r}^{2} \rightarrow \int_{\partial W} \boldsymbol{v} \cdot \boldsymbol{T} \boldsymbol{n} d \mathcal{H}^{n-1}=\int_{\partial W \cap \Omega} \boldsymbol{v} \cdot \boldsymbol{T} \boldsymbol{n} d \mathcal{H}^{n-1}+\int_{\partial W \cap \partial \Omega} \boldsymbol{v} \cdot \boldsymbol{T} \boldsymbol{m} d \mathcal{H}^{n-1}
\end{gathered}
$$

where in (2.20) we use the decomposition $\partial W=(\partial W \cap \Omega) \cup(\partial W \cap \partial \Omega)$ and the equality $\boldsymbol{n}=\boldsymbol{m}$ for $\mathcal{H}^{n-1}$ a.e. point of $\partial W \cap \partial \Omega$. Furthermore, the continuity of $\boldsymbol{v}$ at $\mathbf{0}$ implies

$$
I_{r}^{3} \rightarrow \boldsymbol{v}(\mathbf{0}) \cdot \lim _{r \rightarrow 0} I_{r}^{4}
$$

where

$$
I_{r}^{4}=\int_{W \cap S_{r}} \boldsymbol{T}(\boldsymbol{x}) \boldsymbol{x} / r d \mathcal{H}^{n-1}(\boldsymbol{x})
$$

provided the limit in the right hand side exists. We write $\boldsymbol{T}_{0}$ for $\boldsymbol{T}_{0}(\boldsymbol{x} / r)$ and make the following rearrangements of $I_{r}^{4}$ :

$$
\begin{aligned}
I_{r}^{4}= & \int_{W \cap C \cap S_{r}}\left(\boldsymbol{T}(\boldsymbol{x})-\boldsymbol{T}_{0} r^{-n+1}\right) \boldsymbol{x} / r d \mathcal{H}^{n-1} \\
& +\left(\int_{C \cap S_{r}}-\int_{(C \backslash W) \cap S_{r}}\right) \boldsymbol{T}_{0} r^{-n} \boldsymbol{x} d \mathcal{H}^{n-1} \\
& +\int_{(W \backslash C) \cap S_{r}} \boldsymbol{T}(\boldsymbol{x}) \boldsymbol{x} / r d \mathcal{H}^{n-1}
\end{aligned}
$$

We note that

$$
\int_{W \cap C \cap S_{r}}\left|\boldsymbol{T}(\boldsymbol{x})-\boldsymbol{T}_{0} r^{-n+1}\right| d \mathcal{H}^{n-1} \rightarrow 0
$$

by (2.8). Furthermore,

$$
\int_{C \cap S_{r}} \boldsymbol{T}_{0} r^{-n} \boldsymbol{x} d \mathcal{H}^{n-1}=\int_{C \cap \mathbb{S}^{n-1}} \boldsymbol{T}_{0}(\boldsymbol{d}) \boldsymbol{d} d \mathcal{H}^{n-1}(\boldsymbol{d})=-\boldsymbol{c}
$$

for scaling reasons since $C$ is a cone with vertex $\mathbf{0}$. Next note that since $\boldsymbol{T}_{0}$ is bounded, say by $c$, we have

$$
\left|\int_{(C \backslash W) \cap S_{r}} \boldsymbol{T}_{0} r^{-n} \boldsymbol{x} d \mathcal{H}^{n-1}\right| \leq c r^{-n+1} \mathcal{H}^{n-1}\left((C \backslash W) \cap S_{r}\right) \rightarrow 0
$$




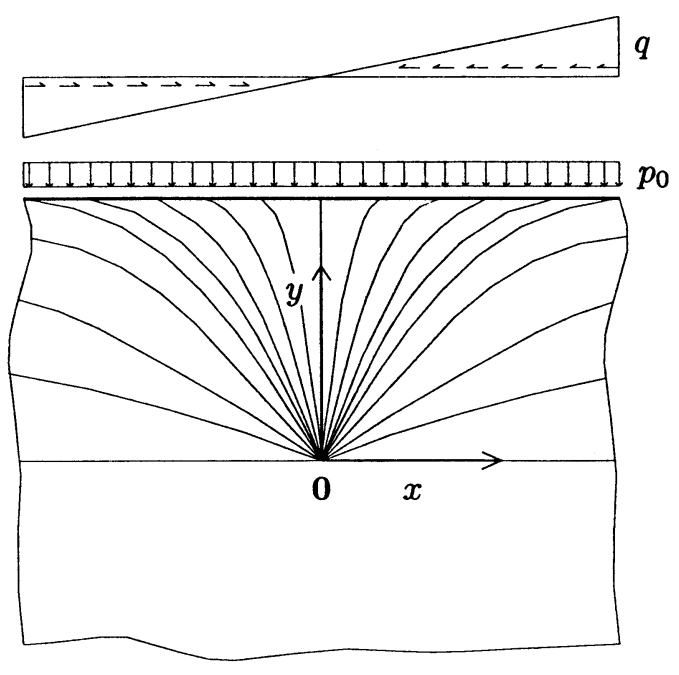

FIG. 1. The isostatic curves for the half-plane.

by (2.7). Since $|\boldsymbol{T}(\boldsymbol{x})| \leq c|\boldsymbol{x}|^{-n+1}$ for every $\boldsymbol{x} \in W \backslash\{\mathbf{0}\}$ and some $c$, a combination with (2.7) provides

$$
\left|\int_{(W \backslash C) \cap S_{r}} \boldsymbol{T}(\boldsymbol{x}) \boldsymbol{x} / r d \mathcal{H}^{n-1}\right| \leq c r^{-n+1} \mathcal{H}^{n-1}\left((W \backslash C) \cap S_{r}\right) \rightarrow 0 .
$$

Thus (2.22) implies

$$
I_{r}^{4} \rightarrow-c
$$

Combining (2.15), (2.18), (2.19), (2.20), (2.21), and (2.23), we find that

$$
\begin{aligned}
\int_{\Omega} \nabla \boldsymbol{v} \cdot d \mathbf{T}= & -\int_{W} \boldsymbol{v} \cdot \operatorname{div} \boldsymbol{T} d \mathcal{L}^{n} \\
& +\int_{\partial W \cap \Omega} \boldsymbol{v} \cdot \boldsymbol{T} \boldsymbol{n} d \mathcal{H}^{n-1} \\
& +\int_{\partial W \cap \partial \Omega} \boldsymbol{v} \cdot \boldsymbol{T} \boldsymbol{m} d \mathcal{H}^{n-1} \\
& -\boldsymbol{v}(\mathbf{0}) \cdot \boldsymbol{c} .
\end{aligned}
$$

Assume that $\mathbf{0} \in \Omega$. Then the measures $\operatorname{div} \boldsymbol{T} \mathcal{L}^{n} \mathbf{L} W, \boldsymbol{T} \boldsymbol{n} \mathcal{H}^{n-1}\left\llcorner(\partial W \cap \Omega),-\boldsymbol{c} \delta_{\mathbf{0}}\right.$ are supported in $\Omega$ and the measure $\boldsymbol{T} \boldsymbol{m} \mathcal{H}^{n-1}\llcorner(\partial W \cap \partial \Omega)$ is supported on $\partial \Omega$; comparing (2.24) with (2.2), we see that $\mathbf{T}$ is an equilibrated tensor field and, moreover, $\operatorname{div} \mathbf{T}$ and $\mathrm{N}(\mathbf{T})$ are given by (2.10) and (2.11). If $\mathbf{0} \in \partial \Omega$, then the measure $-\boldsymbol{c} \delta_{\mathbf{0}}$ is supported on $\partial \Omega$ and a comparison of (2.24) with (2.2) gives (2.13) and (2.14).

3. The half-plane. Let us consider a half-plane $H$ with a horizontal boundary $\partial H$ subjected to its own weight and to distributed normal and tangential loads $p_{0}$ and $q$, respectively, on $\partial H$, where $p_{0}$ is constant and $q$ is a linear function of the horizontal coordinate of the point on $\partial H$; see Fig. 1. 
Suppose that the stress tensor $\boldsymbol{S}$ is negative semidefinite and that its determinant is zero,

$$
\boldsymbol{S} \leq \mathbf{0}, \quad \operatorname{det} \boldsymbol{S}=0 .
$$

The negative semidefiniteness is the basic property of no-tension materials; if the two eigenvalues of $\boldsymbol{S}$ are negative, the material behaves as a linear elastic material [1] while its behavior is trivial if the two eigenvalues vanish; thus in dimension two the main non-trivial case arises if one eigenvalue is negative and the other vanishes; this leads to $\operatorname{det} \boldsymbol{S}=0$. We write

$$
H:=\left\{(x, y) \in \mathbb{R}^{2}: y<y_{0}\right\}
$$

where $y_{0}>0$. Changing the value of $y_{0}$ amounts to shifting the origin of the coordinate system in the vertical direction and thus $y_{0}$ can be chosen arbitrarily; however, later we shall eventually choose $y_{0}$ so as to make the coordinate line $\{(x, 0): x \in \mathbb{R}\}$ coincident with the line where the solution becomes singular. The outer normal to $H$ is $\boldsymbol{e}=(0,1)$, the body force is $\boldsymbol{b}=(0,-b)$ where $b>0$ is the uniformly distributed specific weight of the body, and the normal traction on $\partial H$ is given by

$$
\boldsymbol{t}(\boldsymbol{x})=\left(q^{\prime} x,-p_{0}\right)
$$

for any $\boldsymbol{x}=\left(x, y_{0}\right) \in \partial H$, where $q^{\prime}<0, p_{0}>0$ are constants. With

$$
\boldsymbol{S}=\boldsymbol{S}(x, y)=\left[\begin{array}{ll}
S_{x x} & S_{x y} \\
S_{x y} & S_{y y}
\end{array}\right],
$$

we rewrite (3.1) as

$$
S_{x x} \leq 0, \quad S_{y y} \leq 0, \quad S_{x x} S_{y y}=S_{x y}^{2}
$$

the equilibrium equations $\operatorname{div} \boldsymbol{S}+\boldsymbol{b}=\mathbf{0}$ and $\boldsymbol{S e}=\boldsymbol{t} \mathrm{read}$

$$
\left.\begin{array}{l}
S_{x x, x}+S_{x y, y}=0, \\
S_{x y, x}+S_{y y, y}=b
\end{array}\right\}
$$

and

$$
\left.\begin{array}{l}
S_{x y}\left(x, y_{0}\right)=q^{\prime} x, \\
S_{y y}\left(x, y_{0}\right)=-p_{0} .
\end{array}\right\}
$$

We shall show that the problem admits a unique solution on the strip

$$
\Sigma=\left\{(x, y): x \in \mathbb{R}, 0<y \leq y_{0}\right\}
$$

for which $S_{x y}$ is of the separated form

$$
S_{x y}(x, y)=f(x) g(y)
$$

where $f, g$ are some functions on $\mathbb{R}$ and $\left(0, y_{0}\right)$, respectively. We motivate $(3.5)$ by the relative simplicity of the resulting solution, in particular by the simple form of the active isostatic curves of $\boldsymbol{S}$ to be determined below. Our original derivation of the solution was based on the characteristics of the system (3.3)-(3.4) and on a transformation of variables employed in [14], which in particular suggested the crucial substitution (3.10) (below).

A comparison of (3.5) with the boundary condition (3.4) 1 shows that $S_{x y}$ has the form

$$
S_{x y}=x g, \quad g\left(y_{0}\right)=q^{\prime} .
$$


Inserting $S_{x y}$ into the balance equations, integrating, and comparing the result with the boundary condition $(3.4)_{2}$, we obtain

$$
\begin{gathered}
S_{x x}=-\frac{1}{2} x^{2} g^{\prime}+r, \\
S_{y y}=-G+b\left(y-y_{0}\right)-p_{0}
\end{gathered}
$$

where $G$ is the primitive function of $g$ with $G\left(y_{0}\right)=0$ and $r$ some function of $y$. The determinantal condition $(3.2)_{3}$ reads

$$
\left(\frac{1}{2} x^{2} g^{\prime}-r\right)\left(G+p_{0}-b\left(y-y_{0}\right)\right)=x^{2} g^{2} ;
$$

expanding the product and comparing the coefficients in front of $x$, we obtain

$$
\begin{gathered}
\frac{1}{2} g^{\prime}\left(G+p_{0}-b\left(y-y_{0}\right)\right)=g^{2}, \\
r\left(G+p_{0}-b\left(y-y_{0}\right)\right)=0 .
\end{gathered}
$$

From the last equation we obtain $r=0$ identically. Inserting $r=0$ into (3.7) and using $S_{x x} \leq 0$, we see that $g^{\prime} \geq 0$; since $g\left(y_{0}\right)=q^{\prime}<0$, we deduce that $g<0$ for $y<y_{0}$. The differentiation of (3.8) with respect to $y$ gives

$$
g^{2} g^{\prime \prime}+\frac{1}{2} g^{\prime 2}(g-b)=2 g g^{\prime 2} .
$$

Referring to the negativity of $g$, we now make the substitution

$$
g=-1 / \eta^{2}
$$

where $\eta>0$. The relations

$$
g^{\prime}=\frac{2 \eta^{\prime}}{\eta^{3}}, \quad g^{\prime \prime}=\frac{2 \eta \eta^{\prime \prime}-6 \eta^{2}}{\eta^{4}}
$$

reduce $(3.9)$ to

$$
\eta^{\prime \prime}=b \eta \eta^{2}
$$

which integrates to

$$
\eta^{\prime} \exp \left(-b \eta^{2} / 2\right)=c
$$

where $c$ is a constant. Using (3.7) with $r=0,(3.6)_{1}$, and $(3.2)_{3}$, we find that

$$
\boldsymbol{S}(x, y)=-\left[\begin{array}{cc}
\frac{x^{2} \eta^{\prime}}{\eta^{3}} & \frac{x}{\eta^{2}} \\
\frac{x}{\eta^{2}} & \frac{1}{\eta \eta^{\prime}}
\end{array}\right]
$$

where $\eta$ and $\eta^{\prime}$ are evaluated at $y$. The constant $c$ and the function $\eta$ are determined as follows. Combining the boundary conditions with (3.12), we obtain $p_{0}=1 / \eta\left(y_{0}\right) \eta^{\prime}\left(y_{0}\right), q^{\prime}=$ $-1 / \eta\left(y_{0}\right)^{2}$, from which

$$
\eta\left(y_{0}\right)=1 / \sqrt{-q^{\prime}}, \quad \eta^{\prime}\left(y_{0}\right)=\sqrt{-q^{\prime}} / p_{0}
$$

and hence

$$
c=\sqrt{-q^{\prime}} \exp \left(b / 2 q^{\prime}\right) / p_{0} .
$$


Integrating (3.11) over the interval $\left[y, y_{0}\right]$ and making the substitution $t=\sqrt{b / 2} \eta(u)$, we obtain

$$
\begin{aligned}
c\left(y_{0}-y\right) & =\int_{y}^{y_{0}} \exp \left(-b \eta(u)^{2} / 2\right) \eta^{\prime}(u) d u \\
& =\sqrt{2 / b} \int_{\sqrt{b / 2} \cdot \eta(y)}^{\sqrt{-b / 2 q^{\prime}}} \exp \left(-t^{2}\right) d t \\
& =\sqrt{\pi / 2 b}\left(\phi\left(\sqrt{-b / 2 q^{\prime}}\right)-\phi(\sqrt{b / 2} \eta(y))\right)
\end{aligned}
$$

where $\phi:[0, \infty) \rightarrow[0,1)$ is the error function,

$$
\phi(z)=\frac{2}{\sqrt{\pi}} \int_{0}^{z} \exp \left(-t^{2}\right) d t, \quad z \in \mathbb{R}
$$

with the inverse $\phi^{-1}:[0,1) \rightarrow[0, \infty)$. We now choose

$$
y_{0}=\sqrt{\pi / 2 b} \phi\left(\sqrt{-b / 2 q^{\prime}}\right) / c
$$

and observe that for this value of $y_{0},(3.15)$ reduces to

$$
\phi(\sqrt{b / 2} \eta(y))=c \sqrt{2 b / \pi} y .
$$

If $0<y<y_{0}$, then the right hand side of (3.18) is positive and bounded by $\phi\left(\sqrt{-b / 2 q^{\prime}}\right)<$ 1 ; thus (3.18) can be solved for $\eta=\eta(y)$ which gives

$$
\eta(y)=\sqrt{2 / b} \phi^{-1}(\sqrt{2 b / \pi} c y) .
$$

Equations (3.12) and (3.19) provide an explicit solution of the problem in the strip $0<y<y_{0}$ with the required properties.

From (3.16) one finds that $z / \sqrt{\pi} \leq \phi(z) \leq 2 z / \sqrt{\pi}$ whenever $z \geq 0$ is small enough to satisfy $\exp \left(-z^{2}\right) \geq 1 / 2$. The increasing character of $\phi^{-1}$ then gives $\sqrt{\pi} w / 2 \leq \phi^{-1}(w) \leq$ $\sqrt{\pi} w$ for every sufficiently small $w \geq 0$, which in turn implies

$$
c y \leq \eta(y) \leq 2 c y
$$

for every sufficiently small $y \geq 0$ by (3.19). Furthermore,

$$
\phi^{-1}(w)=\frac{1}{2} \sqrt{\pi} w+O\left(w^{3}\right)
$$

for small $w$ and hence

$$
\eta(y)=c y+o(y), \quad \eta^{\prime}(y)=c+o(1)
$$

as $y \rightarrow 0$ by (3.19) and (3.11). Writing $\boldsymbol{x}=(x, y)$ and inserting this asymptotics into (3.12), we obtain

$$
\boldsymbol{S}(\boldsymbol{x})=\boldsymbol{S}_{0}(\boldsymbol{x})+|\boldsymbol{x}|^{2} \boldsymbol{M}(\boldsymbol{x}) /(\boldsymbol{x} \cdot \boldsymbol{e})^{4}
$$

where

$$
\boldsymbol{S}_{0}(\boldsymbol{x})=\boldsymbol{x} \otimes \boldsymbol{x} / c^{2}(\boldsymbol{x} \cdot \boldsymbol{e})^{3}
$$

for every $\boldsymbol{x} \in \mathbb{R}^{2} \backslash\{\mathbf{0}\}$ and $\boldsymbol{M}$ is a tensor-valued function such that

$$
|\boldsymbol{M}(\boldsymbol{x})| \leq m(\boldsymbol{x} \cdot \boldsymbol{e})
$$

where $m:\left[0, y_{0}\right] \rightarrow[0, \infty)$ is a nondecreasing function with

$$
\lim _{y \rightarrow 0} m(y) / y=0 \text {. }
$$


Note for future use that

$$
\operatorname{div} \boldsymbol{S}_{0}=\mathbf{0}
$$

on $\mathbb{R}^{2} \backslash\{\mathbf{0}\}$.

Abbreviating $\eta=\eta(y), \eta^{\prime}=\eta^{\prime}(y)$, we have $\eta^{\prime} / c=\exp \left(b \eta^{2} / 2\right) \leq 2$ for all sufficiently small $y \geq 0$; combining with $(3.20)_{2}$, we obtain $\eta \eta^{\prime} \leq 4 c^{2} y$ and hence

$$
S_{y y}(x, y)=-1 / \eta \eta^{\prime} \leq-1 / 4 c^{2} y
$$

for all $x \in \mathbb{R}$ and all sufficiently small $y \geq 0$. We deduce from (3.25) that $S_{y y}$ is not integrable on $\Sigma$ and hence $\boldsymbol{S}$ cannot be interpreted as a measure on $\Sigma$; in fact $S_{y y}$ is not even locally integrable on $\Sigma$. However, $\boldsymbol{S}$ can be integrable on subsets of $\Sigma$; in particular we shall see below that $\boldsymbol{S}$ is integrable on sectors of $\Sigma$ delimited by active isostatic curves.

By (3.1), one eigenvalue of $\boldsymbol{S}(x, y)$ vanishes while the other eigenvalue is nonpositive; from (3.12) one finds that the nonvanishing eigenvalue is

$$
\lambda=-\frac{1}{\eta \eta^{\prime}}-\frac{x^{2} \eta^{\prime}}{\eta^{3}}<0
$$

where $\eta$ and $\eta^{\prime}$ are evaluated at $y$; the eigenvector corresponding to this eigenvalue is found to be (proportional to)

$$
\boldsymbol{v}=\boldsymbol{v}(\boldsymbol{x})=\left(v_{x}, v_{y}\right)=\left(x / \eta, 1 / \eta^{\prime}\right) .
$$

The active isostatic curves $\boldsymbol{\kappa}$ are the integral curves of $\boldsymbol{v}$, i.e., solutions of

$$
\frac{d y}{d x}=v_{y} / v_{x}
$$

An integration gives

$$
x=\xi \eta(y), \quad 0 \leq y \leq y_{0},
$$

where $\xi \in \mathbb{R}$ is an integration constant. From $\eta(0)=0$ we see that each $\boldsymbol{\kappa}$ starts at the origin $\mathbf{0}$; putting $y=y_{0}$ and using $(3.13)_{1}$, we see that $\boldsymbol{\kappa}$ intersects $\partial H$ at the point $\left(\xi / \sqrt{-q^{\prime}}, y_{0}\right)$ (see Fig. 1). Using $(3.21)_{1}$, one finds that

$$
x=\xi c y+o(y)
$$

as $y \rightarrow 0$.

Let $\xi>0$ be fixed and put

$$
W=\left\{(x, y): 0<y<y_{0}, 0<x<\xi \eta(y)\right\},
$$

which is the region delimited by the $y$ axis, $\partial H$, and the isostatic curve $\boldsymbol{\kappa}$ of equation (3.26); in fact one has

$$
\partial W=L \cup \kappa \cup R
$$

where

$$
L=\partial W \cap \partial H=\left\{\left(x, y_{0}\right): 0 \leq x \leq \xi / \sqrt{-q^{\prime}}\right\}
$$

and

$$
R=\left\{(0, y): 0 \leq y \leq y_{0}\right\} .
$$

Let $\boldsymbol{T}$ be the restriction of $\boldsymbol{S}$ to $W$. One finds that the normal component of $\boldsymbol{T}$ on $\partial W$ vanishes on $\boldsymbol{\kappa} \cup R$ while on $L$ the normal component is equal to $\boldsymbol{S e}=\boldsymbol{t}$. Let us show that $\boldsymbol{T}$ is $\mathcal{L}^{2}$ integrable on $W$. Writing $\boldsymbol{x}=(x, y)$, using (3.22) and (3.23), and 
noting that $m(y) / y$ is bounded for all sufficiently small $y \geq 0$ by (3.24), we obtain $|\boldsymbol{T}(\boldsymbol{x})| \leq c_{1}|\boldsymbol{x}|^{2} / y^{3}$ for all $\boldsymbol{x} \in W$ and some $c_{1}$. The integrability of $\boldsymbol{T}$ on $W$ then follows from $\int_{W}|\boldsymbol{x}|^{2} / y^{3} d \mathcal{L}^{2}(\boldsymbol{x})<\infty$ which is verified by applying a successive integration with respect to $x$ and $y$ and using $(3.20)_{2}$. Thus

$$
\mathbf{T}=\boldsymbol{T} \mathcal{L}^{2}\llcorner W
$$

is a well defined measure on $\mathbb{R}^{2}$.

Let $\Omega \subset \mathbb{R}^{2}$ be any open set with Lipschitz boundary such that

$$
W \subset \Omega, \quad L \subset \partial \Omega
$$

we now wish to determine the divergence $\operatorname{div} \mathbf{T}$ of $\mathbf{T}$ in $\Omega$ and the normal trace $\mathrm{N}(\mathbf{T})$ of $\mathbf{T}$ on $\partial \Omega$. In this section $\Omega$ is arbitrary (subject to (3.27)); in Section 4 we shall employ the results on $\operatorname{div} \mathbf{T}$ and $\mathrm{N}(\mathbf{T})$ with concrete choices of $\Omega$.

Let us show that $\Omega, W$, and $\boldsymbol{T}$ satisfy the hypotheses of Proposition 2.1. Putting

$$
C=\{(x, y): y>0,0<x<\xi c y\},
$$

one finds that $C \cap \Sigma \subset W$ and

$$
W \backslash C=\left\{(x, y): 0<y<y_{0}, \xi c y<x<\xi \eta(y)\right\}
$$

where we recall $(3.20)_{1}$; using $\eta(y)-c y=o(y)$, one deduces that $(2.7)$ is satisfied. Furthermore, putting

$$
\boldsymbol{T}_{0}(\boldsymbol{d})=\boldsymbol{S}_{0}(\boldsymbol{d})
$$

for every $\boldsymbol{d} \in C \cap \mathbb{S}^{1}$, we verify (2.8) as follows. If $r>0$ and $\boldsymbol{x}=(x, y) \in C \cap \partial \mathbf{B}(r)$, then (3.22) gives

$$
r \boldsymbol{T}(\boldsymbol{x})-\boldsymbol{T}_{0}(\boldsymbol{x} / r)=r\left(\boldsymbol{S}(\boldsymbol{x})-\boldsymbol{S}_{0}(\boldsymbol{x})\right)=r^{3} \boldsymbol{M}(\boldsymbol{x}) / y^{4}
$$

and thus

$$
\left|r \boldsymbol{T}(\boldsymbol{x})-\boldsymbol{T}_{0}(\boldsymbol{x} / r)\right| \leq r^{3} m(y) / y^{4}
$$

by (3.23). We have $y \leq r$ and the definition of $C$ gives $\xi c y \geq x=\sqrt{r^{2}-y^{2}}$ from which $y \geq \alpha r$ where $\alpha=1 / \sqrt{\xi^{2} c^{2}+1}$. Thus

$$
\left|r \boldsymbol{T}(\boldsymbol{x})-\boldsymbol{T}_{0}(\boldsymbol{x} / r)\right| \leq \alpha^{-4} r^{3} m(r) / r^{4}=\alpha^{-4} m(r) / r ;
$$

recalling that $m(r) / r \rightarrow 0$, we obtain (2.8), which completes the verification of the hypothesis of Proposition 2.1. Hence, putting

$$
c=-\int_{C \cap \mathbb{S}^{1}} \boldsymbol{S}_{0}(\boldsymbol{d}) \boldsymbol{d} d \mathcal{H}^{1}(\boldsymbol{d})
$$

and using $\operatorname{div} \boldsymbol{S}=-\boldsymbol{b}$ in $W$, we deduce from (2.10) and (2.11) that

$$
\begin{gathered}
\operatorname{div} \mathbf{T}=-\boldsymbol{b} \mathcal{L}^{2}\left\llcorner W+\boldsymbol{c} \delta_{\mathbf{0}},\right. \\
\mathrm{N}(\mathbf{T})=\boldsymbol{t} \mathcal{H}^{1}\llcorner L
\end{gathered}
$$

if $\mathbf{0} \in \Omega$ while from (2.13) and (2.14) we deduce that

$$
\begin{gathered}
\operatorname{div} \mathbf{T}=-\boldsymbol{b} \mathcal{L}^{2} \mathrm{~L} W, \\
\mathrm{~N}(\mathbf{T})=\boldsymbol{t} \mathcal{H}^{1} \mathrm{~L} L-\boldsymbol{c} \delta_{\mathbf{0}}
\end{gathered}
$$


if $\mathbf{0} \in \partial \Omega$. The constant $\boldsymbol{c}$ can be evaluated as follows. Since $\boldsymbol{S}_{0}$ is divergence free and the normal component of $\boldsymbol{S}_{0}$ on $\partial C$ vanishes, the divergence theorem yields

$$
c=-\int_{P} S_{0}(x) e d \mathcal{H}^{1}(x)
$$

where $P$ is any line segment of the form $P=C \cap Q$ where $Q$ is any horizontal line in $\Sigma$. Taking, e.g., $Q=\partial H$, one obtains $P=\left\{\left(x, y_{0}\right): 0 \leq x \leq \xi c y_{0}\right\}$ and

$$
\boldsymbol{S}_{0}(\boldsymbol{x}) \boldsymbol{e}=\boldsymbol{x} / c^{2} y_{0}^{2}
$$

for each $\boldsymbol{x}=\left(x, y_{0}\right) \in P$; an elementary integration in (3.29) gives

$$
\boldsymbol{c}=-\left(\xi^{2} / 2, \xi / c\right)
$$

4. Rectangular panels. Consider a rectangular panel of base $B$ and height $H$, free from loads on its sides and subjected, besides to its own weight, to various loads on its top.

4.1. Example 1. We start with the case for which the loads at the top are as in Fig. 2 , which coincide with a segment of those of Section 3 if one puts $q^{\prime}=-q_{0} / B$. We place the origin of the coordinate system into the lower left corner of the panel. We shall construct the stress field by gluing part of the solution $\boldsymbol{S}$ determined in Section 3 with some simple solution corresponding to $\boldsymbol{b}=(0,-b)$. We recall the value $y_{0}$ from $(3.17)$ and limit ourselves to the case $y_{0}=H$, which corresponds to the situation when the horizontal load has the maximum value compatible with the equilibrium, given $p_{0}$ and b. By (3.17) and (3.13) the equation $H=y_{0}$ reads

$$
H=p_{0} \sqrt{\pi / 2 b} \phi\left(\sqrt{-b / 2 q^{\prime}}\right) \exp \left(-b / 2 q^{\prime}\right) / \sqrt{-q^{\prime}} ;
$$

given $b, H, p_{0}$, this can be viewed as an implicit equation for $q^{\prime}$; introducing the variable $\mu:=\sqrt{-b / 2 q^{\prime}},(4.1)$ can be rewritten as

$$
\sqrt{\pi} \mu \phi(\mu) \exp \left(\mu^{2}\right)=b H / p_{0} .
$$

The panel is divided into the regions $\Omega_{1}$ and $\Omega_{2}$ by the active isostatic curve $\iota$ of $\boldsymbol{S}$ which connects the origin $\mathbf{0}$ with the upper right corner of the panel. This requirement determines the parameter $\xi$ in (3.26) by $\xi \eta(H)=B$; putting $H=y_{0}$ and using $(3.13)_{1}$, we obtain

$$
\xi=B \sqrt{-q^{\prime}}
$$

Expressing the $y$ coordinate of the point $(x, y) \in \iota$ as a function of $x$, i.e., introducing $\bar{y}:[0, B] \rightarrow[0, H]$ by

$$
x=\xi \eta(\bar{y}(x)), \quad 0 \leq x \leq B
$$

we find from (3.19) that

$$
\bar{y}(x)=\sqrt{\pi / 2 b} \phi(\sqrt{b / 2} x / \xi) / c .
$$

The regions $\Omega_{1}, \Omega_{2}$ are given by

$$
\Omega_{1}=\{(x, y): 0 \leq x \leq B, y>\bar{y}(x)\}, \quad \Omega_{2}=\{(x, y): 0 \leq x \leq B, y<\bar{y}(x)\}
$$



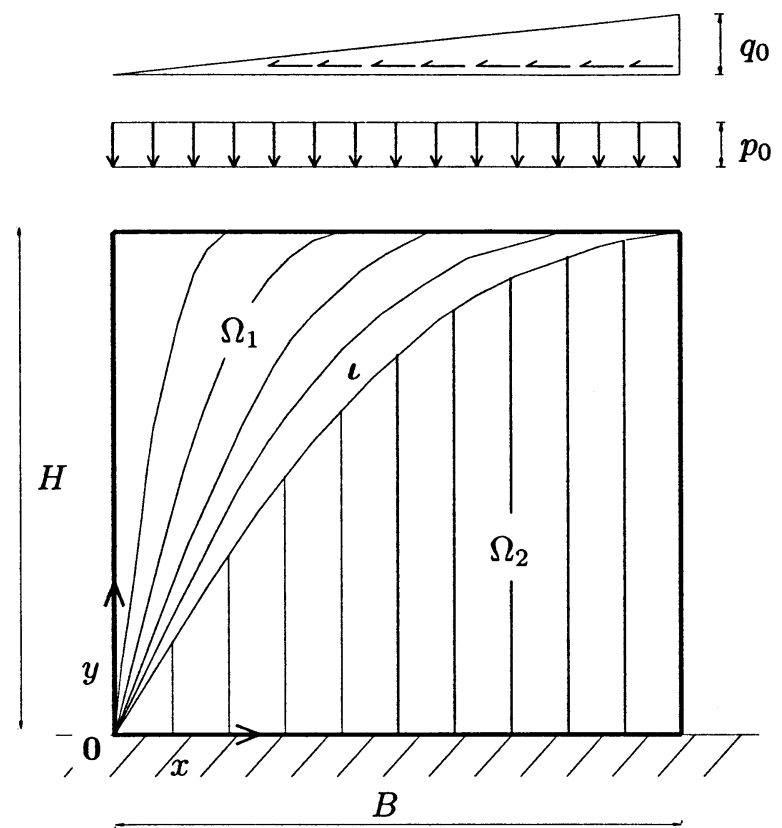

FIG. 2. The rectangular panel with distributed loads on the top.

letting $\boldsymbol{e}=(0,1)$, we put

$$
\boldsymbol{T}(\boldsymbol{x})= \begin{cases}\boldsymbol{S}(\boldsymbol{x}) & \text { if } \boldsymbol{x} \in \Omega_{1}, \\ b(y-\bar{y}(x)) \boldsymbol{e} \otimes \boldsymbol{e} & \text { if } \boldsymbol{x} \in \Omega_{2},\end{cases}
$$

for any $\boldsymbol{x}=(x, y) \in \Omega:=(0, B) \times(0, H)$. Since the classical divergences of the two expressions in (4.4) are equal to $-\boldsymbol{b}$ on $\Omega_{1}, \Omega_{2}$, respectively, and the normal components of these two expressions on $\boldsymbol{\iota}$ vanish, we deduce that the distributional divergence $\mathbf{d i v} \mathbf{T}$ of $\mathbf{T}=\boldsymbol{T} \mathcal{L}^{2} L \Omega$ in $\Omega$ satisfies

$$
\operatorname{div} \mathbf{T}+\boldsymbol{b}=\mathbf{0}
$$

moreover, referring to (3.28), we obtain that the normal trace $\mathrm{N}(\mathbf{T})$ on $\partial \Omega$ is given by

$$
\mathrm{N}(\mathbf{T})=\boldsymbol{t} \mathcal{H}^{1}\left\llcorner\partial \Omega-\boldsymbol{c} \delta_{\mathbf{0}}\right.
$$

where

$$
\boldsymbol{c}=B\left(B q^{\prime} / 2,-p_{0} \exp \left(-b / 2 q^{\prime}\right)\right)
$$

by (3.30), (4.3) and (3.14), and

$$
\boldsymbol{t}(\boldsymbol{x})= \begin{cases}\left(q^{\prime} x,-p_{0}\right) & \text { if } \boldsymbol{x}=(x, H), 0 \leq x \leq B, \\ (0, b \bar{y}(x)) & \text { if } \boldsymbol{x}=(x, 0), 0 \leq x \leq B, \\ \mathbf{0} & \text { otherwise }\end{cases}
$$

for any $\boldsymbol{x} \in \partial \Omega$. We observe that the first component of $\boldsymbol{c}$ equals the resultant of the horizontal load applied to the top of the panel and that the second component of $\boldsymbol{c}$ is 
the sum of the resultant $-B p_{0}$ of the vertical load applied to the top of the panel and the weight of $\Omega_{1}$.

The moment of the body forces in $\Omega_{1}$ with respect to $\mathbf{0}$ is

$$
\begin{aligned}
M_{b} & =-b \int_{\Omega_{1}} x d \mathcal{L}^{2}(\boldsymbol{x}) \\
& =-b \int_{0}^{B} \int_{\bar{y}(x)}^{H} x d y d x \\
& =-b \int_{0}^{B} x(H-\bar{y}(x)) d x \\
& =-b\left(\frac{1}{2} H B^{2}-c^{-1} \int_{0}^{B} x \sqrt{\pi / 2 b} \phi(\sqrt{b / 2} x / \xi) d x\right) ;
\end{aligned}
$$

making the substitution $t=\sqrt{b / 2} x / \xi$, noting that this substitution transforms the upper limit of the last integral to $\mu=\sqrt{-b / 2 q^{\prime}}$, using

$$
\int t \phi(t) d t=\frac{1}{2}\left(t^{2}-\frac{1}{2}\right) \phi(t)+\frac{1}{2 \sqrt{\pi}} t \exp \left(-t^{2}\right)
$$

and employing (4.2), (3.14), and (4.3), we find

$$
M_{b}=\frac{1}{2} B^{2}\left(H q^{\prime}+p_{0}\right) \text {. }
$$

Then it is an easy matter to verify that the region $\Omega_{1}$, when subjected to its weight and the loads applied to the top of the panel, is in equilibrium with respect to the rotation about the point $\mathbf{0}$. Let $r_{m}=q_{0} B / 2$ be the resultant of the horizontal load applied on the top of the panel. Using $\mu:=\sqrt{-b / 2 q^{\prime}}$, we find

$$
r_{m}=\frac{b B^{2}}{4 \mu^{2}}
$$

here $\mu$ can be calculated from the implicit formula (4.2). The graph of $1 / \mu^{2}=4 r_{m} / b B^{2}$ as a function of $\zeta=b H / p_{0}$ is the curve corresponding to $\nu=1$ in Fig. 5 .

4.2. Example 2. Let us consider the case in which the load consists of the uniform vertical load $p=-p_{0}$ on the top of the panel, the weight of the panel, and of a force $\boldsymbol{f}=-\left(f_{1}, f_{2}\right)$ concentrated at the upper right corner of the panel (Fig. 3). For reasons that will become apparent in Example 3, we place the origin of the coordinate system at that corner, with the positive $x$ axis in the right direction and the positive $y$ axis pointing upwards.

Suppose that the stress field is described by a tensor-valued measure $\mathbf{T}$ of the form

$$
\mathbf{T}=\boldsymbol{T}_{r} \mathcal{L}^{2}\left\llcorner\Omega+\boldsymbol{T}_{s} \mathcal{H}^{1}\llcorner\gamma\right.
$$

where $\Omega$ denotes the interior of the panel, $\gamma$ is a curve of equation

$$
y=\omega(x), \quad x_{0} \leq x \leq 0,
$$

with one endpoint $\boldsymbol{x}=\mathbf{0}$ and the other on the base of the panel. Furthermore, $\Omega^{+}$and $\Omega^{-}$are regions resulting from the division of $\Omega$ by $\boldsymbol{\gamma}$, and $\boldsymbol{T}_{r}$ and $\boldsymbol{T}_{s}$ denote the regular 


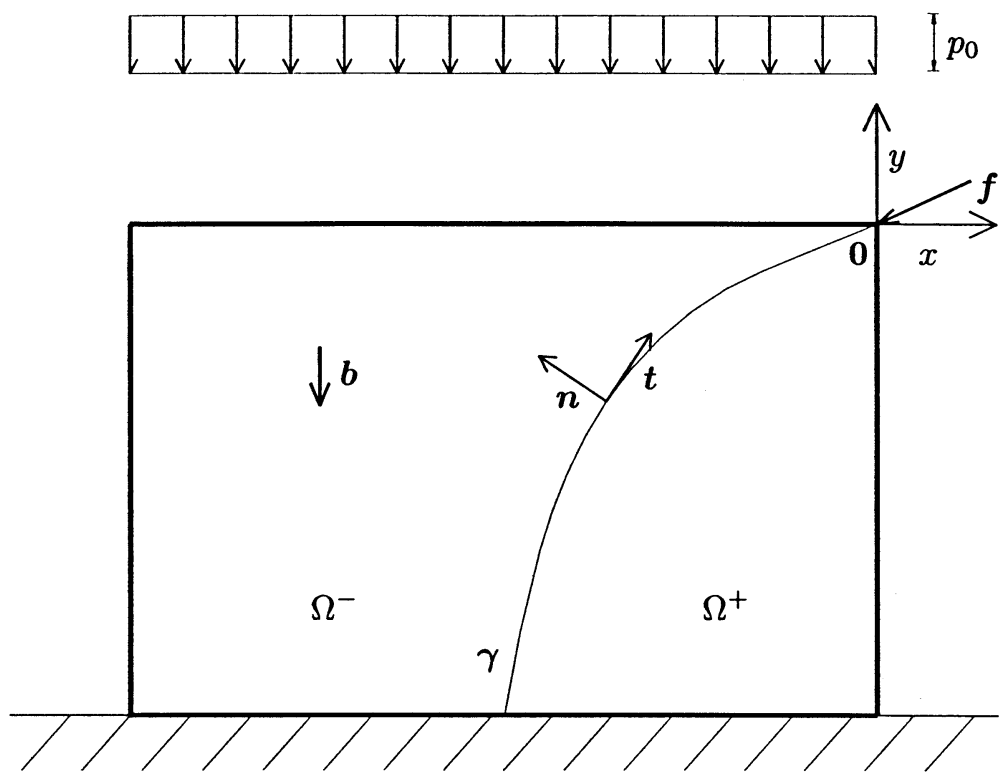

FIG. 3. The rectangular panel undergoing a concentrated force.

and singular parts of the stress. In a way similar to [10] we assume that

$$
\boldsymbol{T}_{r}= \begin{cases}\left(b y-p_{0}\right) \boldsymbol{e} \otimes \boldsymbol{e} & \text { in } \Omega^{-}, \\ b(y-\omega(x)) \boldsymbol{e} \otimes \boldsymbol{e} & \text { in } \Omega^{+},\end{cases}
$$

and observe that $\boldsymbol{T}_{r}$ satisfies (2.5) in $\Omega \backslash \gamma$. The singular stress $\boldsymbol{T}_{s}$ must be superficial; this gives

$$
\boldsymbol{T}_{s}=\sigma \boldsymbol{t} \otimes \boldsymbol{t}
$$

where $\boldsymbol{t}$ is the unit tangent vector to $\gamma$ and necessarily $\sigma \leq 0$ since $\boldsymbol{T}_{s}$ must be negative semidefinite. We determine $\gamma$ and $\sigma$ from (2.6) as follows. Writing

$$
\boldsymbol{t}=J^{-1}\left(1, \omega^{\prime}\right), \quad \boldsymbol{n}=J^{-1}\left(-\omega^{\prime}, 1\right), \quad J:=\sqrt{1+\omega^{\prime 2}},
$$

where the prime denotes the differentiation with respect to $x$, we have [10]

$$
\operatorname{div}_{\gamma} \boldsymbol{T}_{s}=J^{-1}\left((\sigma / J)^{\prime},\left(\sigma \omega^{\prime} / J\right)^{\prime}\right) ;
$$

from (4.11) we obtain

$$
\left[\boldsymbol{T}_{r}\right] \boldsymbol{n}=J^{-1}\left(0, p_{0}-b \omega\right) .
$$

Equation (2.6) then reads

$$
(\sigma / J)^{\prime}=0, \quad\left(\sigma \omega^{\prime} / J\right)^{\prime}=p_{0}-b \omega,
$$

with the boundary condition $\sigma(0) \boldsymbol{t}(0)=\boldsymbol{f}$ at $\boldsymbol{x}=\mathbf{0}$, which gives $\sigma(0) / J(0)=-f_{1}$, $\sigma(0) \omega^{\prime}(0) / J(0)=-f_{2}$ and $\omega(0)=0$; consequently

$$
\sigma(0)=-|\boldsymbol{f}|, \quad \omega^{\prime}(0)=f_{2} / f_{1} .
$$


The solution of (4.12) and (4.13) is

$$
\begin{gathered}
\omega(x)=\alpha \sinh (k x)-\beta \cosh (k x)+\beta, \\
\sigma(x)=-f_{1} \sqrt{1+\omega^{\prime}(x)^{2}}
\end{gathered}
$$

with

$$
k=\sqrt{b / f_{1}}, \quad \alpha=\frac{f_{2}}{\sqrt{f_{1} b}}, \quad \beta=\frac{p_{0}}{b} .
$$

Keeping the direction of $\boldsymbol{f}$ constant, the maximum value of $|\boldsymbol{f}|$ is attained when the curve $\gamma$ meets the lower left corner of the panel. For this value of $|\boldsymbol{f}|$, the region $\Omega^{-}$ is in equilibrium with respect to the rotation around the lower left corner of the panel when $\Omega^{-}$is subjected to its weight, the load $p_{0}$, and the force $\boldsymbol{f}$. The corresponding maximum value $f_{m}$ of the horizontal component $f_{1}$ of $\boldsymbol{f}$ can be determined from the implicit equation

$$
b B^{2}=f_{m}\left(\ln \frac{\sqrt{H b\left(H b+2 p_{0}\right)+b\left(\lambda^{2}-1\right) f_{m}}+H b+p_{0}}{p_{0}+\sqrt{b\left(\lambda^{2}-1\right) f_{m}}}\right)^{2}
$$

where $\lambda=|\boldsymbol{f}| / f_{1}$; that equation is obtained from (4.14) for $x=-B, \omega(x)=-H$. We can verify that if $\lambda^{2}=1+H^{2} / B^{2}$, then the right hand side of (4.16) tends to the left hand side $b B^{2}$ when $f_{m}$ tends to infinity. Thus (4.16) can have a solution only if $\lambda^{2}<1+H^{2} / B^{2}$, i.e., if $f_{2} / f_{1}<H / B$. Moreover for $p_{0}=0$ we deduce that the equilibrium is possible only if $\lambda>1$, that is, if $f_{2} \neq 0$. In particular, if $f_{2}=0$, then $\lambda=1$ and from (4.16) we obtain

$$
f_{m}=\frac{b B^{2}}{\left(\ln \frac{\sqrt{H b\left(H b+2 p_{0}\right)}+H b+p_{0}}{p_{0}}\right)^{2}}
$$

if $p_{0}>0$.

4.3. Example 3. Finally, we consider the uniform vertical load $p_{0}$ on the top and a linear tangential load on a part of the top of width $B_{0}$; see Fig. 4. As in Example 1, the boundary condition partly coincides with that of Section 3 provided we put $q^{\prime}=$ $-q_{0} / B_{0}$, and we shall again use a sector of the solution $\boldsymbol{S}$ of Section 3 delimited by the active isostatic curve $\iota$ that ends in the upper right corner of the panel. We denote by $\Omega=(0, B) \times(0, H)$ the interior of the panel of base $B$ and height $H$, and we place the origin of the coordinate system at the point of singularity of $\boldsymbol{S}$; see Fig. 4. We assume that the stress field is described by a measure $\mathbf{T}$ of the form (4.9) where $\boldsymbol{T}_{r}$ and $\boldsymbol{T}_{s}$ are the regular and singular parts of the stress and $\gamma$ is a curve with one endpoint equal to $\boldsymbol{x}=\mathbf{0}$ and the other endpoint on the base of the panel, given by (4.10).

Let $\iota$ be the isostatic curve of $\boldsymbol{S}$ as in Example 1, let

$$
\boldsymbol{c}:=B_{0}\left(B_{0} q^{\prime} / 2,-p_{0} \exp \left(-b / 2 q^{\prime}\right)\right)
$$




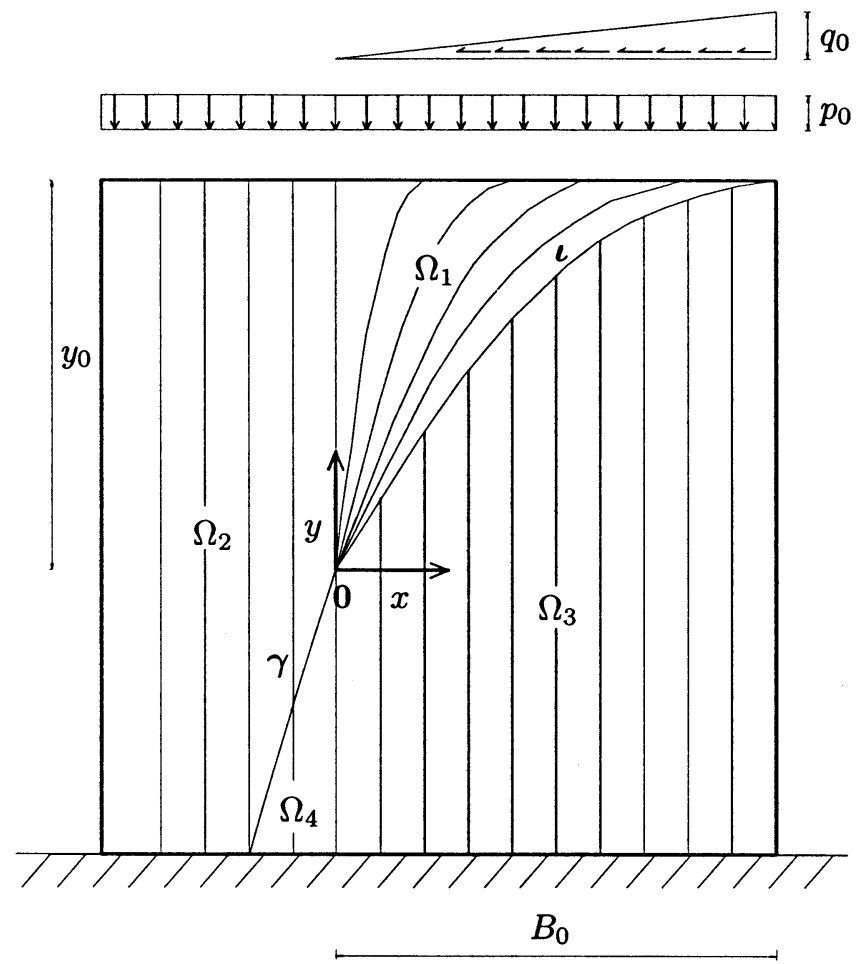

FIG. 4. The isostatic lines for the panel in Example 3.

cf. (4.6) and (4.7), assume that $\boldsymbol{\gamma}$ is given by (4.14) with $\boldsymbol{f}=\boldsymbol{c}$, and define the solution $\mathbf{T}$ of the form (4.9) as follows. Put

$$
\begin{aligned}
& \Omega_{1}=\left\{(x, y): 0<x<B_{0}, y>\bar{y}(x)\right\}, \\
& \Omega_{3}=\left\{(x, y) \in \Omega: 0<x<B_{0}, y<\bar{y}(x)\right\}, \\
& \Omega_{4}=\left\{(x, y) \in \Omega: x_{0}<x<0, y<\omega(x)\right\}, \\
& \Omega_{2}=\Omega \backslash\left(\Omega_{1} \cup \Omega_{3} \cup \Omega_{4}\right),
\end{aligned}
$$

and define the regular part of the stress by

$$
\boldsymbol{T}_{r}(\boldsymbol{x})= \begin{cases}\boldsymbol{S}(\boldsymbol{x}) & \text { if } \boldsymbol{x} \in \Omega_{1}, \\ \left(b\left(y-y_{0}\right)-p_{0}\right) \boldsymbol{e} \otimes \boldsymbol{e} & \text { if } \boldsymbol{x}=(x, y) \in \Omega_{2}, \\ b(y-\bar{y}) \boldsymbol{e} \otimes \boldsymbol{e} & \text { if } \boldsymbol{x}=(x, y) \in \Omega_{3}, \\ b(y-\omega(x)) \boldsymbol{e} \otimes \boldsymbol{e} & \text { if } \boldsymbol{x}=(x, y) \in \Omega_{4}\end{cases}
$$

and the singular part of the stress by

$$
\boldsymbol{T}_{s}=\sigma \boldsymbol{t} \otimes \boldsymbol{t}
$$

where $\boldsymbol{t}$ is the unit tangent vector to $\boldsymbol{\gamma}$ and $\sigma$ is given by (4.15) with $\boldsymbol{f}=\boldsymbol{c}$. Noting that the measure $\mathbf{T}$ coincides with that of Example 1 in the quadrant $\{(x, y), x>0, y>0\}$ and with that of Example 2 with $p_{0}$ redefined to $p_{0}+b y_{0}$ in the quadrant $\{(x, y), x<0, y<0\}$ 


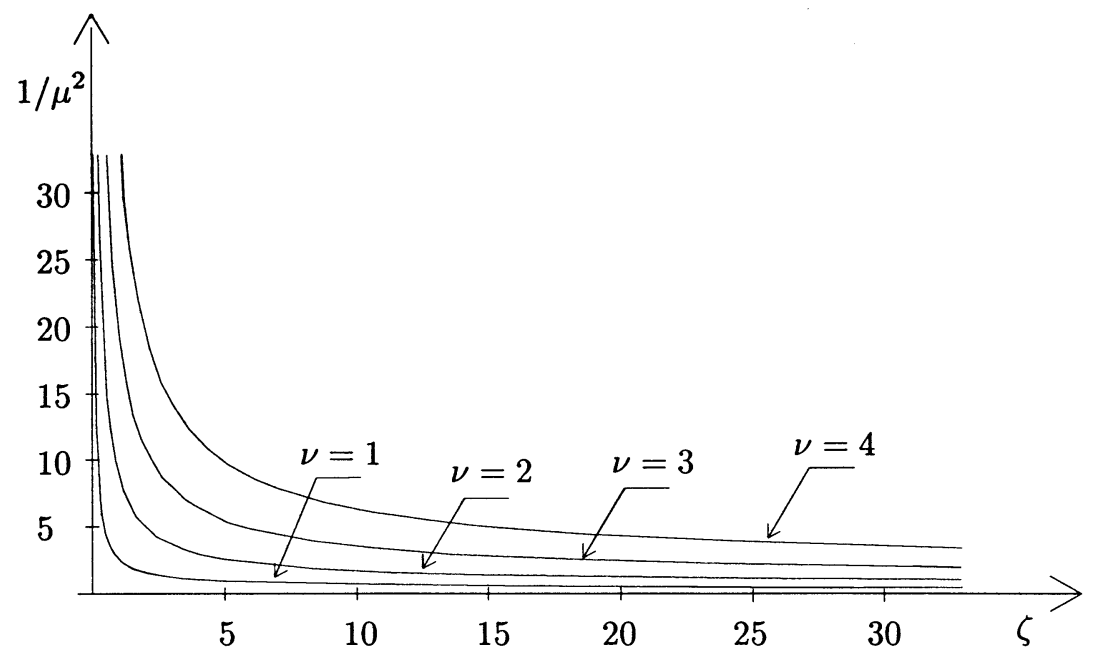

FIG. 5. The behaviour of $1 / \mu^{2}$ as a function of $\zeta$, for different values of $\nu$.

and comparing the expression for normal trace in (4.6) with the boundary conditions in Example 2, it is not hard to see that $\mathbf{T}$ satisfies (4.5) in $\Omega$ and the boundary condition on the top of the panel.

The curve $\gamma$ meets the lower left corner of the panel if $\omega\left(B_{0}-B\right)=y_{0}-H$. Inserting $\omega$ from (4.14) and $y_{0}$ from (3.17), we obtain the implicit relation

$$
\begin{aligned}
\frac{b H}{p_{0}}=\sqrt{\pi} \mu \phi(\mu) \exp \left(\mu^{2}\right) & +\left(\mu \exp \left(\mu^{2}\right)+1 / 2\right) \exp (2 \mu(\nu-1)) \\
& -\left(\mu \exp \left(\mu^{2}\right)-1 / 2\right) \exp (2 \mu(1-\nu))-1,
\end{aligned}
$$

where $\mu=\sqrt{-b / 2 q^{\prime}}$ and $\nu=B / B_{0}$. We note that (4.18) reduces to (4.2) for $\nu=1$. Let $r_{m}$ be the value of the resultant of the horizontal load $B_{0} q_{0} / 2$; taking into account that

$$
r_{m}=\frac{b B_{0}^{2}}{4 \mu^{2}}
$$

by (4.8), we can calculate $r_{m}$ by solving (4.18). Furthermore, one can consider the limit $B_{0} \rightarrow 0$ keeping $r_{m}$ constant. It is easy to see that then the solution of the present example reduces to that of Example 2 with $f_{2}=0$. In particular, the regions $\Omega_{1}$ and $\Omega_{3}$ vanish and (4.18) reduces to (4.17) provided we identify $f_{m} \equiv r_{m}$, as is natural. Fig. 5 shows the graphs of $1 / \mu^{2}=4 r_{m} / b B_{0}^{2}$ versus $\zeta=b H / p_{0}$ for different values of $\nu$.

\section{REFERENCES}

[1] Del Piero, G. Constitutive equations and compatibility of the external loads for linear elastic masonry-like materials. Meccanica, 24 (1989) 150-162. MR1037468 (91i:73004)

[2] Del Piero, G. Limit analysis and no-tension materials. Int. J. Plasticity, 14 (1998) 259-271.

[3] Di Pasquale, S. Statica dei solidi murari teorie ed esperienze. (1984) Dipartimento di Costruzioni, Università di Firenze, Pubblicazione n. 27.

[4] Federer, H. Geometric measure theory. Springer, New York, 1969. MR0257325 (41:1976) 
[5] Gurtin, M. E. An introduction to continuum mechanics. Academic Press, Boston, 1981. MR636255 (84c:73001)

[6] Heyman, J. The stone skeleton. Internat. J. Solids Structures, 2 (1966) 249-279.

[7] Lucchesi, M.; Šilhavý, M.; Zani, N. Singular equilibrated stress fields for no-tension panels. Lecture notes in applied and computational mechanics, vol. 23. Springer, 2005, 255-265.

[8] Lucchesi, M.; Šilhavý, M.; Zani, N. Stress state for heavy masonry panels. Proceedings of the "Colloquium Lagrangianum," Venezia, 2004. Springer (to appear).

[9] Lucchesi, M.; Šilhavý, M.; Zani, N. Stress fields for axisymmetric no-tension bodies. Proceedings of $\mathrm{XVII}^{\text {th }}$ AIMETA Congress, Florence, 2005.

[10] Lucchesi, M.; Šilhavý, M.; Zani, N. A new class of equilibrated stress fields for no-tension bodies. Journal of Mechanics of Materials and Structures, 1 (2006) 503-539.

[11] Lucchesi, M.; Zani, N. On the collapse of masonry panel. Proceedings of VII ${ }^{\text {th }}$ International Seminar on Structural Masonry for Developing Countries, Belo Horizonte, Brazil, 2002.

[12] Lucchesi, M.; Zani, N. Some explicit solutions to equilibrium problem for masonry like bodies. Structural Engineering and Mechanics, 16 (2003) 295-316.

[13] Lucchesi, M.; Zani, N. Stati di sforzo per pannelli costituiti da materiale non resistente a trazione. Proceedings of XVI ${ }^{t h}$ AIMETA Congress, Ferrara, 2003.

[14] Polito, L. Equivalenti lineari di equazioni non lineari alle derivate parziali e loro soluzione con dati iniziali. 2001. ADIA 2001-6, Dipartimento di Ingegneria Aerospaziale, Università di Pisa

[15] Šilhavý, M. Cauchy's stress theorem and tensor fields with divergences in $L^{p}$. Arch. Rational Mech. Anal., 116 (1991) 223-255. MR1132761 (93b:73001)

[16] Šilhavý, M. The Mechanics and Thermodynamics of Continuous Media. Springer, Berlin, 1997. MR1423807 (98j:73001)

[17] Šilhavý, M. Normal traces of divergence measure vectorfields on fractal boundaries. 2005. (Preprint, Dipartimento di Matematica, University of Pisa, October 2005.) MR2168979 (2007b:74001) 